\title{
Experimental investigation on steady granular flows interacting with an obstacle down an inclined channel: study of the dead zone upstream from the obstacle. Application to interaction between dense snow avalanches and defence structures
}

\author{
T. Faug, P. Lachamp, and M. Naaim \\ CEMAGREF ETNA, Domaine Universitaire, BP 76, F-38402 Saint-Martin-d'Hères Cedex, France
}

Received: 20 September 2001 - Accepted: 20 December 2001

\begin{abstract}
An experimental investigation with dry granular flows passing over an obstacle down a rough inclined channel has been performed. The aim is to improve our understanding of the interaction between dense snow avalanches and defence structures. Specific attention was directed to the study of the zone of influence upstream from the obstacle, linked to the formation of a dead zone. The dead zone length $L$ was systematically measured as a function of the obstacle height $H$ and the channel inclination $\theta$, for several discharges. In a whole range of channel inclinations, all the data are shown to collapse into a single curve when properly scaled. The scaling is based on the introduction of a theoretical deposit length (depending on $H, \theta$ and the internal friction angle of the material, $\varphi$ ) and a Froude number of the flow depending on the obstacle height.
\end{abstract}

\section{Introduction}

Effects of defence structures on dense snow avalanches are not widely known. Our approach consists in making a physical micro-scale analysis using physical instrumented models to quantify zones of influence and momentum losses created by obstacles. This physical micro-scale analysis aims at establishing a singularity law for a simple case (2-D steady flow interacting with a single obstacle of simple geometry). It will allow us to validate numerical models which will be used to develop more complex singularity laws in application to the interactions between dense snow avalanches (3-D unsteady flows) and protection structures (complex obstacle geometries).

We first made experiments on model material. Until now it is primarily the similarity with dry granular flows which has been exploited: sand (Chu et al., 1995), beads (Hutter et al., 1995), ping-pong balls (Keller et al., 1998). A dense snow avalanche is generally constituted of particles with various

Correspondence to: T. Faug (thierry.faug@ cemagref.fr) forms, sizes and densities. Among the cases most usually encountered, one can distinguish between:

- new snow avalanches (the particles diameter ranging from $1 \mathrm{~mm}$ to $1 \mathrm{~cm}$ ),

- avalanches of snowballs, agglomerated by capillary action or compaction (their diameter ranging from $1 \mathrm{~cm}$ to $1 \mathrm{~m})$ and

- avalanches of angular blocks coming from remainders of snow cover dislocation.

In these three cases, one can compare snow to a granular material. However, this choice runs up against the significant role of water and consequently, against the changing character of moving snow. If this choice is very debatable for wet snow flows, it seems to be reasonable for snow in cold and dry weather.

The present paper reports an investigation of the effects of a weir on granular flows down an inclined channel. We focus our attention on the zone of influence upstream of the obstacle linked to the formation of a dead zone. First, we will describe the experimental device and the granular material used. The following part will present the experimental data. Finally, we will discuss the results we obtained.

\section{Experimental set-up and procedures}

For our tests, we used glass beads, with an average diameter of $300 \mu \mathrm{m}$. The internal friction angle of this material is $\varphi=27^{\circ} \pm 0.5^{\circ}$. The density of glass beads is $\rho_{p}=2460 \mathrm{~kg} \cdot \mathrm{m}^{-3}$. As illustrated in Fig. 1, the experimental set-up consisted of a 2-m-long Plexiglas channel, two hoppers, and a pumping system. We put glass plates $(2 \mathrm{~mm}$ thickness) on vertical walls to limit the electrostatic effects and to preserve the good transparency of the sidewalls. The working width of the channel was thus reduced to $46 \mathrm{~mm}$. The roughness on the channel bottom was made by gluing beads (with the same diameter as beads used for flow) on 


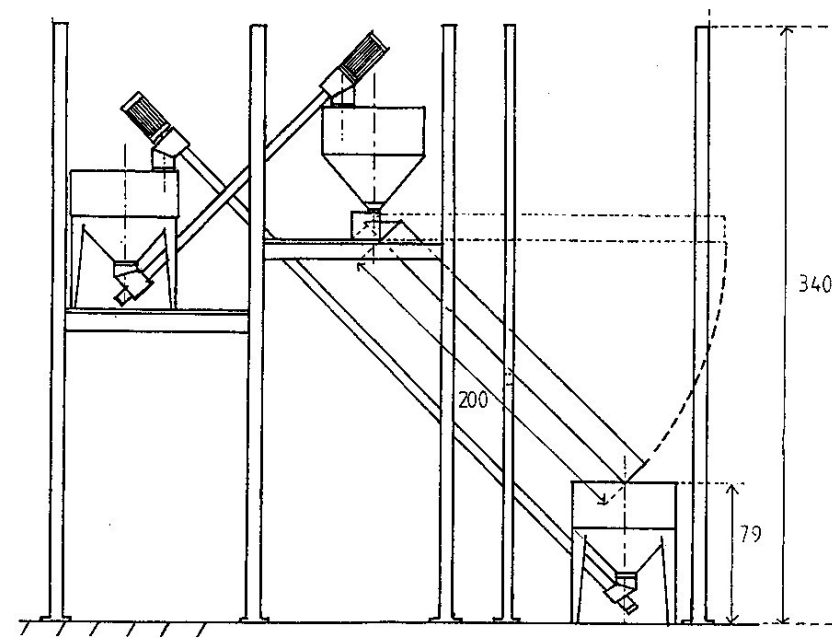

Fig. 1. Schematic view of the experimental device.

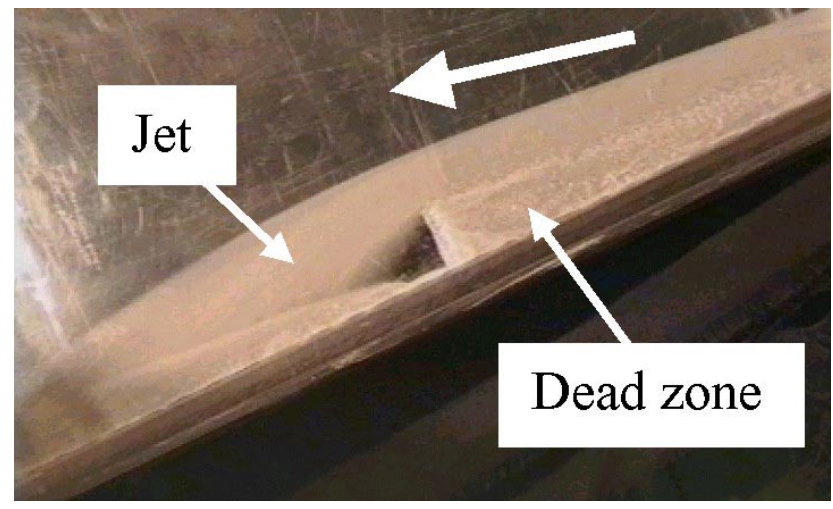

Fig. 2. Photograph of the dead zone and the jet.

a thin PVC band (1 mm thickness, $2 \mathrm{~m}$ length). The technique involved depositing beads on a double-sided adhesive film. The pumping system allows us to let circulate the material without interruption. We used rough bed in order to have steady uniform flows in a whole range of channel inclinations. Indeed, studies on granular flows down rough inclined channels (Savage, 1984; Ancey et al., 1996) or planes (Pouliquen, 1999) showed the existence of steady uniform flows in a whole range of inclination angles, between a value closed to the internal friction angle of the material and a critical angle depending on the type of granular material (approximately $34^{\circ}-35^{\circ}$ for glass beads in our tests).

To control the mass flow rate, the supply hopper at the upper channel entrance was provided with a cone-shaped valve which more or less obstructed the hopper exit. The material flowed down the channel and fell into a collection hopper. Screws powered by two Siemens gears returned the material to the supply hopper. The cone-shaped valve was calibrated by weighing the mass of fallen material for a given interval of time. As is well known for hoppers, the mass flow rate is independent of the height of material stocked inside it; it usually depends only on the size of the particles and the exit

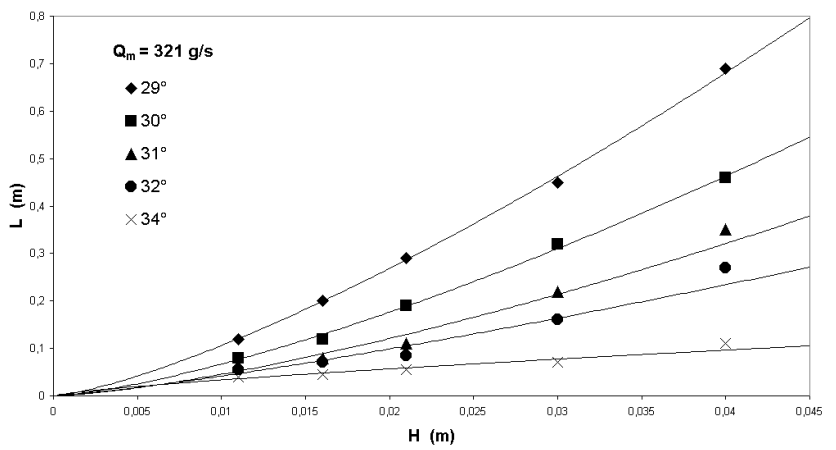

Fig. 3. Dead zone length versus obstacle height for different channel slopes $\left(Q m=321\right.$ g.s $\left.{ }^{-1}\right)$.

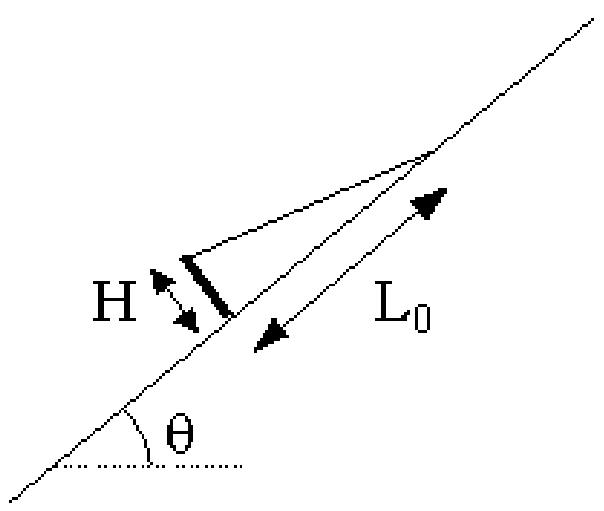

Fig. 4. Schematic view of the deposit.

geometry. Uncertainty on the mass flow rate was estimated at around $5 \%$.

For the reference flows (without obstacle), flow depths were measured with a video camera. Uncertainty on the flow depth was estimated at around $0,5 \mathrm{~mm}$. The discharge $Q$ was deduced from the mass flow rate $Q_{m}$ by assuming that the density was nearly uniform for a steady flow: $Q_{m}=\phi \rho_{p} Q$, with $\phi$ the solid concentration assumed constant. The mean velocity was computed as: $u=q / h$, where $q=Q / l$ is the discharge per unit width, $h$ the flow depth and $l$ the channel width.

\section{Experimental data}

\subsection{Observed phenomena}

A weir was placed in the channel. We tested several obstacle heights ranging from $10 \mathrm{~mm}$ to $40 \mathrm{~mm}$. When the granular flow interacts with the weir, we observe two main features (Fig. 2): the formation of a dead zone upstream from the obstacle and the formation of a jet downstream from the obstacle. Before making measurements into the jet, we first focus our attention on the dead zone because we think that it governs the jet features and energy dissipations. 


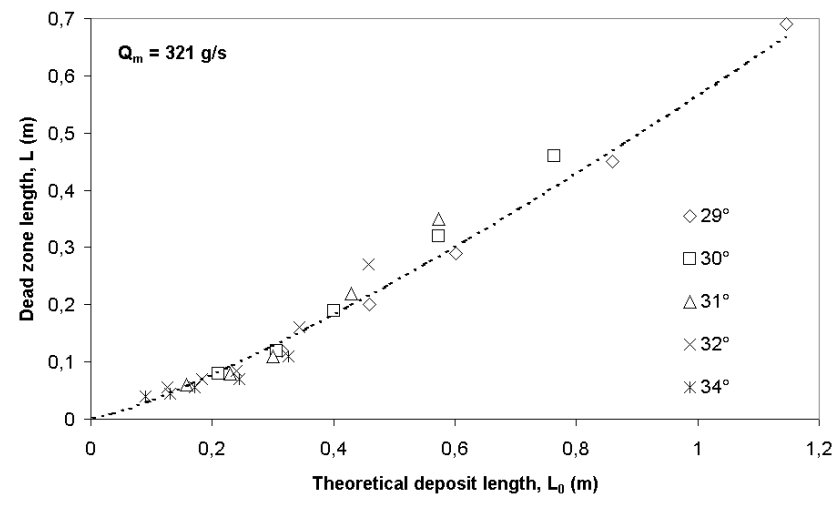

Fig. 5. Dead zone length versus deposit theoretical length for different channel slopes $\left(Q m=321 \mathrm{~g} . \mathrm{s}^{-1}\right)$.

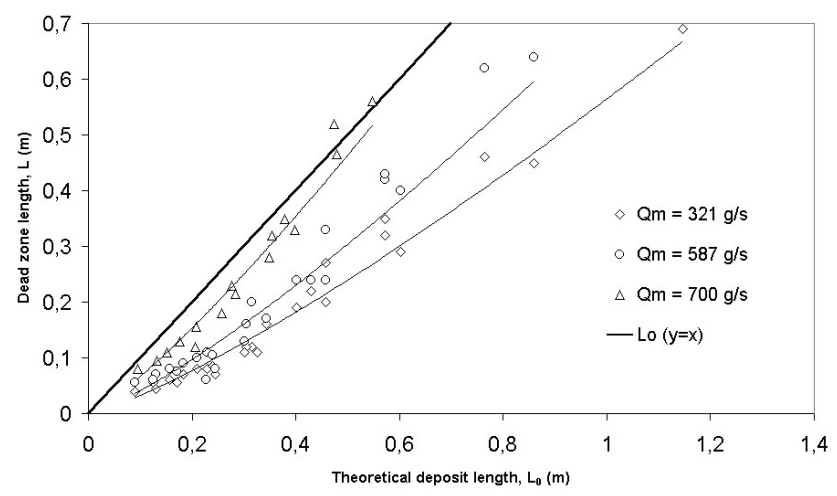

Fig. 6. Dead zone length versus deposit theoretical length for different mass flow rates.

\subsection{Influence of the obstacle height and the channel slope}

In Fig. 3, the dead zone length $L$ is plotted as a function of the obstacle height $H$ for different inclination angles $\theta$ and a mass flow rate set to $321 \mathrm{~g} . \mathrm{s}^{-1}$.

We obtained straight lines with slope depending on the channel inclination. An appropriated dimensional analysis allows us to group the data into a single curve. We introduced the length $L_{0}$ of a theoretical deposition upstream from the obstacle when the flow stops. If we assume that the deposit is equal to the internal friction angle of the granular material, the deposit length $L_{0}$ (Fig. 4) is found to be:

$L_{0}=\frac{H}{\tan (\theta-\varphi)}$.

In Fig. 5, we plotted the dead zone length according to this deposit length for a mass flow rate set to $321 \mathrm{~g} . \mathrm{s}^{-1}$. Others mass flow rates were tested and exactly the same dimensional analysis could be performed (Fig. 6).

In Fig. 6, we also reported the straight line $y=x(L=$ $\left.L_{0}\right)$. We can notice that the dead zone length tends to $L_{0}$ when the mass flow rate increases. This phenomenon could be explained by the transition (depending on a critical mass flow rate) between two different regimes: a frictional one for which $L$ tends to $L_{0}$ (low speed and high flow depth) and a

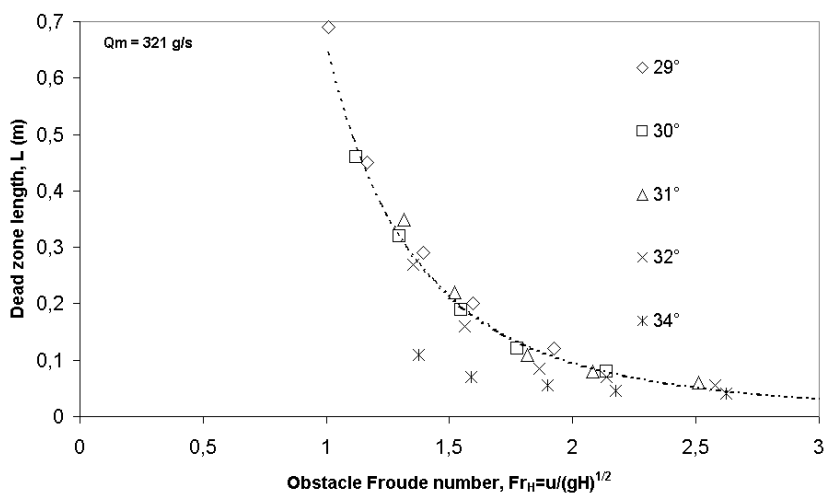

Fig. 7. Dead zone length versus the obstacle Froude number $(\mathrm{Qm}=$ 321 g.s ${ }^{-1}$ ).

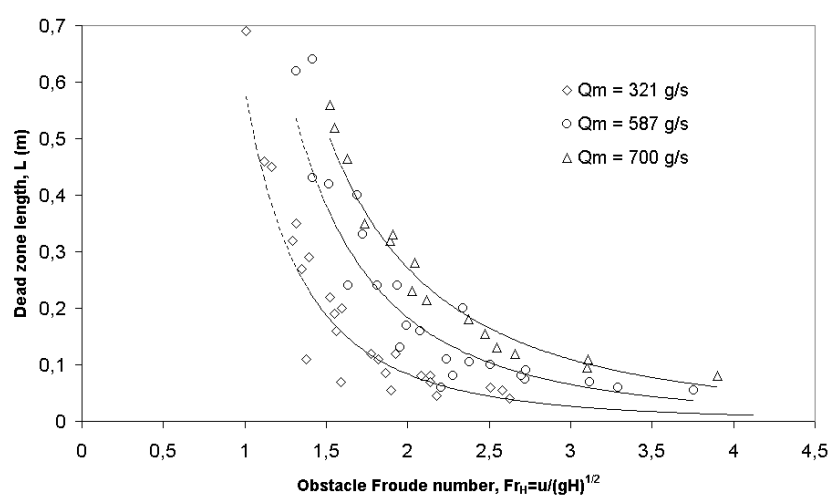

Fig. 8. Dead zone length versus the obstacle Froude number.

collision regime for which $L<L_{0}$ (high speed and low flow depth).

\subsection{Influence of flow velocity and depth}

Considering $L_{0}$, we only take into account the channel and obstacle geometries $(\theta$ and $H$ ), and the intrinsic characteristics of the granular material $(\varphi)$, but the ratio $L / L_{0}$ depends on flow velocity and depth. To consider the reference flow, a classical way is to introduce the Froude number of the flow: $F r=u /(g h)^{1 / 2}$, where $u$ is the mean velocity and $h$ the flow depth. We couldn't find a link between the ratio $L / L_{0}$ and the Froude number. Instead of considering the internal Froude number of the flow, if we consider the obstacle Froude number, we obtained an interesting correlation (Fig. 7). The obstacle Froude number is calculated as follows:

$F r_{H}=\frac{u}{\sqrt{g H}}=\frac{q}{h \sqrt{g H}}$.

A similar dimensional analysis leads to the same results for the other mass flow rates (Fig. 8).

\subsection{Empirical law}

Figures 6 and 8 lead to the following results: 


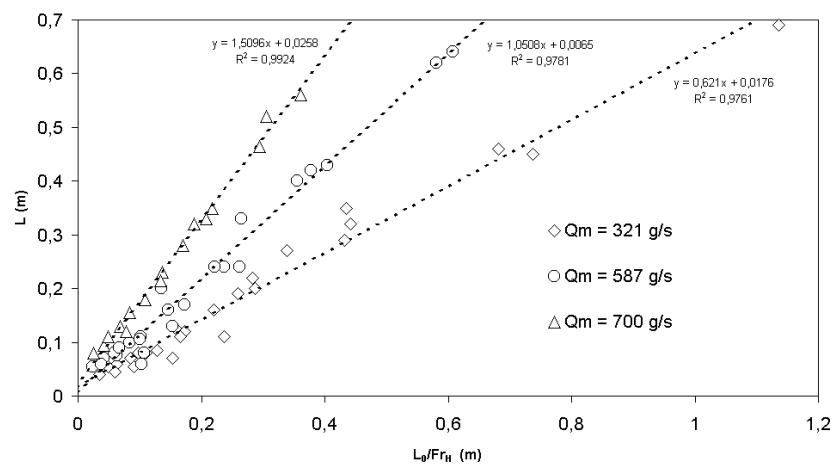

Fig. 9. Dead zone length versus the ratio of the deposit length to the obstacle Froude number.

- the dead zone length $L$ is strongly linked to a theoretical deposit length $L_{0}$ calculated as follows: $L_{0}=H / \tan (\theta-\varphi)$;

- the dead zone length $L$ is strongly linked to the obstacle Froude number $\mathrm{Fr}_{H}$ calculated as follows: $F r_{H}=u /(g H)^{1 / 2}$, with $u=q / h$. The dead zone length vanishes when the obstacle Froude number is high and it increases very rapidly when the obstacle Froude number is close to 1 .

Starting from these results, it appears logical to study the variations of the dead zone length according to the ratio $L_{0} / F r_{H}$. In the tested range of slopes, the experimental observations lead to the following scaling: for a given mass flow rate, the dead zone length varies linearly with the ratio of a theoretical deposit length to the obstacle Froude number (Fig. 9):

$L=\alpha \frac{L_{0}}{F r_{H}}$,

with $\alpha$ a dimensionless parameter depending on the discharge.

Equation (3) could be also written:

$\frac{L}{h}=\alpha(q) \frac{H^{3 / 2}}{\tan (\theta-\varphi)} \frac{\sqrt{g}}{q}$.

Notice that, in Fig. 9, in the range of tested mass flow rates, the slope of each line increases with the mass flow rate. Therefore, in order to find an equation not depending on the discharge per unit width $q$, the first idea is to choose the dimensionless parameter $\alpha$ such as: $\alpha(q) \propto \frac{q}{d^{3 / 2} \sqrt{g}}$, in which $d$ is a characteristic length. By substituting $\alpha$ in Eq. (4), we finally obtain:

$$
\frac{L}{h}=\frac{\beta}{\tan (\theta-\varphi)} H^{3 / 2} \text { with } \beta \propto d^{-3 / 2} \text {. }
$$

We reported in Fig. 10 the ratio $L / h$ as a function of $\theta$ and $H$. All the measurements are shown to collapse into a single curve.

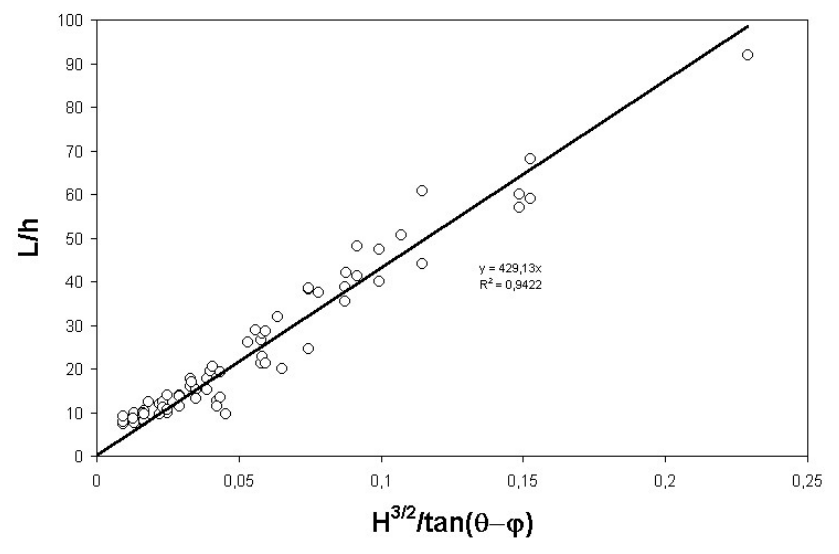

Fig. 10. $L / h$ as a function of $H^{3 / 2} / \tan (\theta-\varphi)$.

\section{Discussion and conclusion}

In this paper, we have experimentally studied the effects of an obstacle on steady granular flows and particularly the formation of a dead zone upstream from the obstacle. By systematically studying the variation of the dead zone length with the obstacle height, the channel slope and the flow depth, we have obtained an interesting scaling. For a given discharge, all the data are shown to collapse into a straight line. The dead zone length varies linearly with the ratio of a theoretical deposit length according to the obstacle Froude number:

$L \propto \frac{L_{0}(H, \theta, \varphi)}{F r_{H}(u, H)}$.

Notice that this scaling has been obtained for a range of Froude numbers of the reference flow $\operatorname{Fr}(u, h)$ between 2 and 6. It would be interesting to test lower Froude numbers. Furthermore, in our experiments the ratio of the obstacle height $H$ to the flow depth $h$ was generally between 1 and 7 (only three values were under 1:0.68, 0.83 and 0.98 ). It would be interesting to test lower values of this ratio. But the system we use currently (not enough quantity of material, limited channel length) does not allow us to test these values.

This scaling predicts a $3 / 2$ power law for the variation of $L$ with $H$, for a constant inclination channel. Furthermore, it shows that $L$ varies linearly with $h$. So we studied the variations of the ratio $L / h$ according to $H^{3 / 2} / \tan (\theta-\varphi$ ) for all the measurements (without mass flow rate influence). All the data are shown to collapse into a straight line. To nondimensionalize the data, it is necessary to introduce a characteristic length, but our experiments cannot provide any idea about it. We keep in mind two important parameters that are constant in our experiments: the particle diameter and the channel width.

However, this scaling provides an empirical way to predict the dead zone length. It will present a great interest to be able to estimate energy dissipations downstream from the obstacle. Indeed, the knowledge of the dead zone length allows us to estimate the mean slope of the dead zone and, therefore, the direction of the velocity at the jet onset (just above 
the obstacle). Starting from these measurements we will be able to study the jet and estimate energy dissipations. Future work will concern the following points:

- the formation of the dead zone (evolution of $L$ as a function of the time);

- the jet will be also studied (steady flow and evolution of the jet length as a function of the time);

- the influence of the particle diameter and the walleffects (influence of the channel width).

\section{References}

Ancey, C., Coussot, P., and Evesque, P.: Examination of the possibility of a fluid-mechanics treatment of dense granular flows,
Mech. Cohesive-Frictional Materials, 1, 385-403, 1996.

Chu, T., Hill, G., McClung, D. M., Ngun, R., and Sherkat, R.: Experiments on granular flows to predict avalanche runup, Can. Geotech. J., 32, 285-295, 1995.

Hutter, K., Koch, T., Plüss, C., and Savage, S. B.: The dynamics of avalanches of granular materials from initiation to runout. Part II: Experiments, Acta Mechanica, 109, 127-165, 1995.

Keller, S., Ito, Y., and Nishimura, K.: Measurements of the velocity distribution in ping-pong-ball avalanches, Annals of Glaciology, 26, 259-264, 1998.

Pouliquen, O.: Scaling laws in granular flows down rough inclined planes, Phys. Fluids, 11, 542-548, 1999.

Savage, S. B.: The mechanics of rapid granular flows, Advances in applied mechanics, 24, 289-366, 1984. 